\title{
Sarcopenia: abordaje integral del adulto mayor
}

\author{
Sarcopenia: integrated care approaches for older people
}

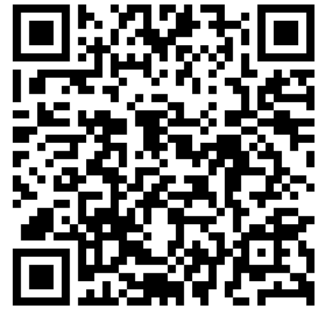

${ }^{1}$ Médico general, graduado de Universidad de Ciencias Médicas (UCIMED). Código médico: 16387

crojabermudez@gmail.com

${ }^{2}$ Médico general, graduado de la Universidad de

Ciencias Médicas (UCIMED). Código médico: 15288. aldabuckv@gmail.com.

${ }^{3}$ Médico General, graduada de Universidad de Ciencias Médicas (UCIMED).Código médico: 15329. magabrielabj@gmail.com.

\author{
${ }^{1}$ Dr. Christopher Rojas Bermúdez \\ Investigador independiente, San José, Costa Rica \\ crojabermudez@gmail.com \\ https://orcid.org/0000-0001-7277-8565
}

${ }^{2}$ Dr. Aldair Buckcanan Vargas

Soporte Vital, Alajuela, Costa Rica aldabuckv@gmail.com

https://orcid.org/0000-0001-6031-1474

${ }^{3}$ Dra. Gabriela Benavides Jiménez Hospital Clínica Bíblica, San José, Costa Rica magabrielabj@gmail.com

https://orcid.org/0000-0002-5206-3965

$\begin{array}{lcc}\text { RECIBIDO } & \text { CORREGIDO } & \text { ACEPTADO } \\ 18 / 03 / 2019 & 30 / 03 / 2019 & 06 / 04 / 2019\end{array}$

\section{RESUMEN}

Sarcopenia es una enfermedad musculo esquelética asociada a la edad con pérdida de la masa y función muscular. Es una patología de gran prevalencia en la población adulta mayor. Su etiología es multifactorial donde influye la nutrición, estilo de vida y factores hormonales. Se recomienda tamizar a todo adulto por encima de los 65 años con el cuestionario SARC-F. Su diagnóstico es probable si se demuestra una pérdida de fuerza muscular, ya sea mediante la prueba de fuerza agarre mano o la prueba de levantarse de una silla sin apoyo de las manos. Se confirma el diagnóstico si a esto se le añade una prueba que demuestre baja masa muscular (para lo cual DXA es el más recomendado) o en la calidad muscular. Es un caso severo al presentarse una prueba de la marcha menor a $0.8 \mathrm{~m} / \mathrm{s}$. Actualmente no existe ningún fármaco aprobado para su uso, por lo que el manejo se basa en entrenamiento de resistencia y aumento de ingesta proteica.

PALABRAS CLAVES: sarcopenia; obesidad; marcha; desnutrición; fragilidad; fuerza muscular.

\section{ABSTRACT}

Sarcopenia is an age related musculoeskeletal disease with loss of the muscular mass and its function. It is a pathology with great prevalence among the elderly. The etiology is multifactorial where nutrition, life style 
and hormones have an influence. Screenig is recomended on all adults at age of 65 years or older with the SARCF questionnaire. The diagnosis is probable if there is a loss on muscle strenght demonstrated on hand grip or a chair stand test. Diagnosis is made when you add a test that shows low muscle mass (DXA is the recommendation) o low muscle quality. The severe cases are the ones that present with a gait speed test below 0.8 meters/seg. Actually, there is no drug aproved for its use, so management is based on resistance trainig and a higher protein intake.

KEYWORDS: sarcopenia; obesity; gait; malnutrition; fragility; muscular strength.

\section{INTRODUCCIÓN}

La sarcopenia es una enfermedad musculo esquelética con deterioro a nivel de la función y cantidad de la masa muscular que tiene una alta prevalencia en el adulto mayor. Aumenta la mortalidad en este grupo etario además de asociarse con múltiples comorbilidades y complicaciones que repercuten en la calidad vida. No existe tratamiento farmacológico especifico aprobado, sino que manejo es con enfoque nutricional y de rehabilitación física. Debido a ser una condición común en medicina general y especializada es necesario tener claro su abordaje. Se realiza una revisión de las guías internacionales y literatura reciente con enfoque en conceptos básicos, tamizaje, diagnóstico y terapéutica con el de objetivo de aumentar la sospecha clínica de la patología en la práctica médica diaria para así poder realizar diagnósticos tempranos que ayuden a brindar un manejo integral.

\section{DEFINICIÓN}

El término sarcopenia fue descrito por primera vez a finales de los años ochenta como una pérdida progresiva de la masa muscular esquelética asociada al envejecimiento.

Actualmente se reconoce que no sólo se encuentra una menor cantidad de tejido muscular, sino que también existe un deterioro en la calidad, fuerza y rendimiento muscular. The International Clinical Practice Guidelines for Sarcopenia (ICFSR) (1) la define como una enfermedad musculoesquelética asociada a la edad que se destaca por perdida de la masa muscular y su función. A partir del 2016 la Organización Mundial de la Salud (OMS) incluye a la sarcopenia como enfermedad en su Clasificación Internacional de Enfermedades y Problemas Relacionados con la Salud (CIE) con el código ICD-10-CM (M62.84) (2).

The European Working Group on Sarcopenia in Older People (EWGSOP2) (3) en su última actualización en 2018 destaca que se asocia con mortalidad, riesgo de caídas, fracturas y disminución capacidad física. Este grupo utiliza como parámetro primario la cantidad de fuerza muscular. Se reconoce que es el mejor parámetro para predecir efectos adversos en comparación con la masa muscular, además es un indicador de función muscular. El rendimiento físico ayuda a predecir la severidad de la sarcopenia. 


\section{CLASIFICACIONES}

- Sarcopenia primaria: Relacionada con la edad y envejecimiento. No se encuentra ningún otro causante.

- Sarcopenia secundaria: Cuando ocurre debido a una enfermedad sistémica principalmente enfermedades que involucren procesos inflamatorios como neoplasias o falla orgánica. Otras causas pueden ser ingesta inadecuada, anorexia y malabsorción.

- Sarcopenia aguda: Duración menor a 6 meses. Usualmente relacionada a una enfermedad aguda secundaria,

- Sarcopenia crónica:Tiene una duración mayor a 6 meses. Asociado a enfermedades progresivas $y$ crónicas. Representa mayor riesgo de mortalidad.

\section{EPIDEMIOLOGÍA}

La prevalencia de la enfermedad es de un $6-22 \%$ de la población por encima de los 65 años de edad (1), de un 14-38\% para aquellos que residen en hogares de adulto mayor y de $10 \%$ de los hospitalizados (2). La evidencia es clara en que la prevalencia aumenta conforme la edad, sin embargo, no parece existir alguna diferencia epidemiológica entre géneros.

\section{ETIOLOGÍA}

La sarcopenia es producto de una combinación de causas genéticas, fisiológicas y de factores ambientales.

Es considerada una parte inevitable del envejecimiento, no obstante, el grado de sarcopenia es variable y depende de la presencia de ciertos factores de riesgo (4).

\section{Deficiencia nutricional}

Es común la reducción de ingesta de alimentos con la edad, debido a pérdida sentido del gusto, olfato, pobre salud oral, vaciamiento gástrico lento, niveles elevados de colecictoquinina, demencia, depresión y el ambiente social.

Se cree que la ingesta de proteínas en exceso en la cantidad para mantener el balance del nitrógeno es necesaria, la recomendación es al menos $1.2 \mathrm{~g} / \mathrm{kg}$ de peso (5). La disminución de la ingesta calórica y de proteínas en la vejez contribuyen a la atrofia muscular y a la severidad de la sarcopenia (6).

\section{Estilo de vida sin ejercicio}

La inactividad física y el desuso muscular promueve la pérdida de masa muscular y empeora el grado de sarcopenia, siendo el factor de riesgo principal. En adultos mayores sanos, tan solo 10 días de encamamiento resultan en la pérdida de la fuerza en las piernas, potencia y capacidad aeróbica (5).

Una disminución gradual del número de fibras musculares inicia a los 50 años (4), esta disminución de fibras musculares al igual que la disminución de la fuerza es mayor en personas sedentarias en comparación con personas más activas; incluso en atletas profesionales se muestra una disminución gradual, aunque más lenta, en la velocidad y fuerza con el envejecimiento (4).

\section{Desequilibrio de hormonas y citoquinas}

La pérdida muscular extrema a menudo resulta de una combinación de la 
disminución de señales hormonales anabólicas y de un aumento de señales catabólicas mediadas por citoquinas proinflamatorias, como el factor de necrosis tumoral a (TNFa) y la Interleukina 6 (IL-6), niveles elevados de ambas se han visto presentes en el músculo esquelético de los adultos mayores (4). Evidencia considerable indica que el envejecimiento altera las concentraciones circulantes de hormonas importantes para el desarrollo muscular (6), incluye disminución en la hormona del crecimiento $(\mathrm{GH})$, la testosterona, la hormona tiroidea y el factor de crecimiento similar a la insulina, llevan a la pérdida de masa muscular y fuerza (4). Dos hormonas anabólicas importantes son:

a) Testosterona: estudios indican que el nivel de testosterona libre disminuye aproximadamente un 3\% por año entre las edades de 73 y 94 años (6), además disminuye la sensibilidad en los tejidos diana, como el músculo.

b) Hormona del crecimiento (GH): es importante en el crecimiento $y$ mantenimiento de la masa muscular, estudios revelan que los niveles circulantes disminuyen aproximadamente un $50 \%$ entre los 20 y 70 años (6).

\section{Metabolismo de proteínas}

El equilibrio entre la descomposición de las proteínas y las tasas de síntesis es crucial para mantener la masa muscular y los estudios demuestran que existe una relación directa con la sarcopenia, se encontró que la síntesis de proteína muscular mixta se redujo en un $30 \%$ con la edad avanzada (6). Las proteínas oxidadas aumentan en el músculo esquelético con el envejecimiento y llevan a la acumulación de lipofuscina y proteínas reticuladas que se eliminan de forma inadecuada a través del sistema de proteólisis, esto conduce a una acumulación de proteína disfuncional no contráctil en el músculo esquelético, y es parte de la razón por la cual la fuerza muscular disminuye severamente en la sarcopenia (4).

\section{Remodelación de la unidad motora}

También ocurre una reducción de las células nerviosas motoras responsables de enviar señales del cerebro hacia los músculos conforme a la edad (4). Con estudios de observación microscópica directa, se vio que el número de neuronas motoras en la médula espinal disminuye con el envejecimiento, concomitantemente el aumento del tamaño de las unidades motoras restantes y la pérdida de fibras musculares también se han identificado como factores contribuyentes de sarcopenia (6).

\section{Base evolutiva}

Las teorías evolutivas implican el fracaso del cuerpo para mantener la masa muscular y función con el envejecimiento, esta hipótesis sugiere que los genes para realizar altos niveles de esfuerzo muscular obligatorio, que eran requeridos para la supervivencia en la época del Paleolítico Tardío, no coinciden con un estilo de vida moderno sedentario (4).

\section{Influencias tempranas del desarrollo}

El bajo peso al nacer, un marcador de un ambiente precoz deficiente, se asocia con una reducción de la masa muscular y la fuerza en la vida adulta, un estudio demostró que un menor peso al nacer se asocia con una disminución significativa 
en la puntuación de fibra muscular (4), lo que sugiere que las influencias del desarrollo en la morfología muscular pueden explicar la asociación entre el bajo peso al nacer y la sarcopenia.

\section{HISTOPATOLOGÍA}

La sarcopenia temprana se da por una disminución en el tamaño del músculo y con el tiempo también se produce una reducción en la calidad del tejido muscular. Los cambios se caracterizan por la sustitución de las fibras musculares por tejido adiposo, aumento de la fibrosis, cambios en el metabolismo muscular, estrés oxidativo y degeneración de la unión neuromuscular (4); que, en última instancia, conduce a una pérdida progresiva de la función muscular y a la fragilidad. Estudios en mecanismos moleculares indican que la patogénesis sugiere que la masa y la función muscular normal depende de un balance dinámico entre reguladores positivos (proteínas morfogénicas del hueso (BMP), factor neurotrófico derivado del cerebro (BDNF), folistatina (FST), irisina) y negativos (factor de crecimiento transformante $\beta$ (TGF $\beta$ ), miostatina, activinas $A$ y $B$, factor de diferenciación del crecimiento (GDF15) del crecimiento muscular (5).

Cambios histológicos en las fibras musculares revelan que la sarcopenia afecta predominantemente a las fibras musculares tipo II (contracción rápida), mientras que las fibras tipo I (contracción lenta) se ven mucho menos afectadas. El tamaño de las fibras tipo II puede reducirse hasta un $50 \%$ en sarcopenia, sin embargo, esas reducciones son moderadas en comparación con las reducciones globales en la masa muscular (4). Esto se relaciona a que la sarcopenia represente una reducción en el número de fibras musculares como en el tamaño de las fibras.

\section{BIOMARCADORES}

En la sarcopenia y la fragilidad física se han propuesto el estudio de biomarcadores, sin embargo, por la variedad de procesos patogénicos que contribuyen al desarrollo y progresión, así como la naturaleza sindrómica de los dos términos y el amplio espectro de fenotipos, conllevan a un reto para la identificación de marcadores biológicos específicos y refleja la ausencia de biomarcadores fidedignos.

Los biomarcadores circulantes más populares son los relacionados a la respuesta inflamatoria (PCR, IL-6 y TNFa), a los parámetros clínicos ( $\mathrm{Hb}$, albumina sérica), hormonas (dehidroepiandrosterona, testosterona, factor de crecimiento similar a la insulina 1 y vitamina D), productos del daño oxidativo o antioxidantes (7). Otros utilizados son los telómeros de las células mononucleares en la circulación periférica, el péptido N-terminal procolágeno tipo III (P3NP), el fragmento c-terminal del agrin (CAF), el eHsp72, las miokinas (miostatina, factor inhibidor de leucemia, IL-6, IL-7, factor neurotrófico cerebral, factor similar a la insulina 1, factor de crecimiento fibroblástico 2, proteína relacionada a folistatina $1 \mathrm{e}$ irisina) y la creatinina-D3.

Hasta el momento no se ha encontrado un único marcador biológico que siga confiablemente a las múltiples contribuyentes y fenotipos de la fragilidad física y sarcopenia (7), ya que existen 
otros factores contribuyentes como el ambiente, que puede ser el gatillo para iniciar los mecanismos fisiopatológicos.

\section{TAMIZAJE.}

La ICFSR (1) recomienda un tamizaje anual a todo adulto por encima de los 65 años y también que se realice en esta población posterior a un evento de salud mayor, como por ejemplo una hospitalización por un infarto agudo miocardio. Se recomienda también tamizar a pacientes que reportan síntomas o signos de sarcopenia los cuales son caídas, debilidad, marcha lenta, dificultar para levantase de una silla, perdida peso y emaciación (8). En la actualidad existen 2 método aceptados de tamizaje, los cuales son el cuestionario SARC-F (A Simple Questionnaire to Rapidly Diagnose Sarcopenia) (9) y valorar la velocidad de la marcha.

EWGSOP2 y ICFSR recomiendan aplicar el SARC-F. Se puede utilizar a nivel de atención primaria. Se basa en 5 preguntas que reportan los pacientes basado en sus propias percepciones sobre sus limitaciones en ámbitos como capacidad para caminar, levantarse de una silla, subir escaleras, también sobre su experiencia en caídas. Presenta una bajamoderada sensibilidad y alta especificidad. Debidos a estar característica, la mayor de cantidad de casos que se captarán serán los severos. Un puntaje $\geq 4$ debe incentivar a realizar estudios complementarios (10).

LA ICFSR recomienda también la velocidad de la marcha, aunque se podría considerar más como una herramienta más bien diagnostica al igual que la fuerza de agarre.

\section{DIAGNÓSTICO}

La EWGSOP2 (3) define que sarcopenia es probable si existe baja fuerza muscular y se confirma el diagnostico si a esto se le añade la comprobación de baja cantidad / calidad muscular. Cuando existen las 3 variables juntas (baja cantidad/calidad muscular, bajo rendimiento físico y baja fuerza muscular) la enfermedad debe ser considerada severa. Ver TABLA 1.

\begin{tabular}{|c|c|c|}
\hline $\begin{array}{c}\text { Probable } \\
\text { sarcopenia }\end{array}$ & Sarcopenia & $\begin{array}{l}\text { Sarcopenia } \\
\text { Severa }\end{array}$ \\
\hline \multirow[t]{3}{*}{$\begin{array}{l}\text { Baja fuerza } \\
\text { muscular }\end{array}$} & $\begin{array}{l}\text { Baja fuerza } \\
\text { muscular }\end{array}$ & $\begin{array}{l}\text { Baja fuerza } \\
\text { muscular }\end{array}$ \\
\hline & $\begin{array}{l}\text { Baja cantidad / } \\
\text { calidad muscular }\end{array}$ & $\begin{array}{l}\text { Baja cantidad/ } \\
\text { calidad } \\
\text { muscular }\end{array}$ \\
\hline & & $\begin{array}{l}\text { Bajo } \\
\text { rendimiento } \\
\text { físico }\end{array}$ \\
\hline \multicolumn{3}{|c|}{$\begin{array}{l}\text { Nota: Datos adaptados según el consenso europeo para } \\
\text { definición y diagnóstico de sarcopenia. } \\
\text { Fuente: Cruz-Jentoft AJ, Bahat G, Bauer J, Boirie Y, Bruyère } \\
\text { O, Cederholm T, et al. Sarcopenia: revised European consensus } \\
\text { on definition and diagnosis }\end{array}$} \\
\hline
\end{tabular}

\section{HERRAMIENTAS Y MÉTODOS DIAGNÓSTICOS}

Se evalúan todos los componentes de sarcopenia: fuerza muscular y masa / calidad muscular además del rendimiento físico que implica a la severidad de la enfermedad.

Existen muchas técnicas diagnósticas, por lo que se mencionaran y explicaran solo las que tiene mayor evidencia.

\section{- Fuerza muscular}

- Prueba fuerza de agarre de las manos: barato y simple. Útil en 
atención primaria. Predictor de efectos adversos como estadías más largas en hospitales, limitaciones funcionales, mala calidad vida y muerte (8). Para obtener una medida exacta se requiere un dinamómetro calibrado. En caso de no poder realzar la prueba por tener la mano deshabilitada como ocurre en artritis o posterior a un evento cerebrovascular se puede utilizar métodos isométricos con torque en extremidades inferiores. Se define como una fuerza menor a $<27 \mathrm{~kg}$ en hombres y $<16 \mathrm{~kg}$ en mujeres (8).

- Prueba fuerza al levantarse de una silla: evalúa fuerza muscular de los cuádriceps.

Se evalúa al medir la cantidad de tiempo necesario para que un paciente se levante 5 veces de su asiento desde la posición de sentado sin utilizar las manos como apoyo.

El valor corte es de 15 segundos (8).

Existen otras variantes que evalúan la cantidad de veces que se levanta en un tiempo ya estimado.

Ambas pruebas son recomendadas por múltiples guías.

\section{- Masa muscular}

Se recomienda el uso del DXA (Dual energy $\mathrm{X}$-ray absorptiometry) para valorar la cantidad de masa magra (1). La mayoría de guías internacionales recomiendan su uso $(1,2,8)$. Una de sus principales limitantes es que no mide la masa muscular del troco y dorso (11) y la deshidratación afecta su resultado. La resonancia magnética y TAC son el estándar de oro para medir la composición corporal pero debido a su costo, la radiación y dificultad de acceso no son tan útiles en la práctica clínica diaria. Los valores corte diagnósticos del DXA para la masa esquelética apendicular (MEA) son un radio MEA/Altura $<7.0 \mathrm{~kg} / \mathrm{m} 2$ en hombres y $<6.0 \mathrm{~kg} / \mathrm{m} 2$ en mujeres (8).

Si ninguno de estos 3 métodos está a disposición, se le sugiere al clínico que utilice su juicio médico para valorar la masa muscular. Otra opción es el uso de la impedancia bioeléctrica. También se encuentran los valores antropométricos. La antropometría no es un buen parámetro para masa muscular, aun así, se ha demostrado que la circunferencia de la pantorrilla (valor corte $<31 \mathrm{~cm}$ ) predice superveniencia y rendimiento en adultos mayores (8), por lo cual es una opción para valorar pronostico en sitios donde no existe ninguna técnica diagnostica.

El ultrasonido muscular es una técnica que viene en aumento. Permite valorar el grosor muscular, área de sección, grosor de los fascículos y ecogenicidad, esta última refleja la funcionabilidad. La pérdida de tejido contráctil e infiltración grasa muscular se muestran como hiperecoicas.

\section{- Calidad muscular}

Se analizan cambios microscópicos y macroscópicos en la arquitectura y composición muscular. No existe consenso sobe métodos de análisis. Se ha utilizado la resonancia magnética y TAC para evaluar la calidad muscular al determinar por ejemplo infiltración grasa 
en musculo. Es un área que se espera tenga gran desarrollo en el futuro.

\section{- Rendimiento físico}

Su evaluación es una medida objetiva de toda la función corporal relacionada a la locomoción (8).

Se evalúa el sistema nervioso central y periférico además del área muscular. Hay casos donde no es posible obtener una medida certera como por ejemplo pacientes con trastornos de la marcha 0 balance y demencia.

- Pruebas de velocidad de la marcha: Es rápida, segura y confiable. De las más utilizadas. Predice efectos adversos y mortalidad. La más utilizada es la marcha de 4 metros, en donde se mide con tiempo la rapidez. Un corte de velocidad menor a $\leq 0.8$ $\mathrm{m} / \mathrm{s}$ para ambos sexos indica sarcopenia severa (8)

- Caminata de $\mathbf{4 0 0}$ metros: Técnicamente difícil de aplicar en atención primaria Se considera diagnostica si no completa la prueba o dura $\geq 6 \mathrm{~min}$.

- The Short Physical Performance Battery (SPPB)

- Prueba de levanta y anda (the Timed-Up and Go test (TUG)

Estas últimas dos pruebas, son combinaciones de pruebas de marcha, de levantarse de la silla y balance. La EWGSOP2 y IFCRS recomiendan la prueba de velocidad de la marcha debido a que no ocupa gran espacio para aplicarla y su facilidad técnica, los cuales son aspectos a valorar sobre todo si se está valorando en atención primaria.

Una manera sencilla de abordaje diagnósticos es al realizarla en 4 pasos:
1. Identificar individuos de alto riesgo: Con el cuestionario SARC-F o por sospecha clínica debido a sintomatología / signos.

2. Valorar evidencia de sarcopenia probable: Con la prueba de fuerza de agarre de la mano o la de levantarse de la silla.

3. Confirmar diagnóstico: Evidenciar baja cantidad / calidad muscular con DXA.

4. Determinar severidad: Con la prueba velocidad e la marcha (elección), otra opción es la prueba de marcha de 400 metros.

\section{TRATAMIENTO}

\section{- Tratamiento no farmacológico}

Se ha asociado la inactividad física con pérdida de fuerza y masa muscular (4), por lo tanto, un régimen de ejercicios es la piedra angular del tratamiento de la sarcopenia. Ejercicio de resistencia ha demostrado un aumento en la síntesis de proteínas a nivel muscular a corto plazo (12). Entrenamiento de resistencia y fuerza han demostrado ser efectivos para prevención y tratamiento de sarcopenia, en especial el de resistencia que influye en el sistema neuromuscular aumentando las concentraciones y taza de producción de proteína (13). La intervención con ejercicio debe durar como mínimo 3 meses para lograr un impacto en la función muscular (10).

El uso de suplementos naturales carece de evidencia científica en cuando a eficiencia, interacciones y efectos adversos. Una dieta alta en proteína (1,2$1,6 \mathrm{~g} / \mathrm{kg} /$ día) se recomienda para prevenir sarcopenia asociada a la edad (5). Se debe consumir calorías de 24 a 36 a 
$\mathrm{kcal} / \mathrm{kg} / \mathrm{día}$ y mantener los niveles de vitamina $\mathrm{D}$ alrededor de $40 \mathrm{ng} / \mathrm{ml}$. (14).

- Tratamiento farmacológico

Actualmente la United States Food and Drug Administration (FDA) no ha aprobado ningún fármaco para el tratamiento de sarcopenia. En cuanto al uso de hormona del crecimiento este aumenta la síntesis de proteína y masa muscular pero no así la capacidad funcional.

Se ha investigado el uso de testosterona y esteroides anabólicos, sin embargo, han tenido un modesto efecto positivo y su uso es limitado debido a efectos adversos como riesgo elevado de cáncer de próstata en hombres, virilización en mujeres y riesgo cardiovascular aumentado en ambos sexos.

El MT-102 es el primer agente transformante anabólico catabólico, recientemente se ha utilizado en un estudio clínico fase dos para el tratamiento de caquexia en pacientes con cáncer en estadios avanzados. Demostró aumento significativo del peso en pacientes que recibieron 10mg de MT-102 dos veces al día por 16 semanas comparado con una disminución del peso en pacientes que recibieron placebo (15). Se realizó un estudio en el cual se le administró una cardiotoxina a ratones en el músculo tibial anterior y posterior a 19 días de la administración de esta y en tratamiento con Losartan, se demostró que los ratones que recibieron el fármaco tenían mejor arquitectura y menor fibrosis muscular, comparado contra placebo (16). Lo dicho anteriormente es muy interesante ya que puede servir de modelo base para nuevos agentes terapéuticos.

\section{COMPLICACIONES}

Sarcopenia se asocia a menudo con una condición médica subyacente, siendo imperativo tratar esta condición de fondo antes de la sarcopenia como tal. La caquexia por ejemplo se caracteriza por una severa pérdida de músculo que va de la mano con enfermedades como cáncer, miocardiopatías, e insuficiencia renal en estadios finales. Se ha definido como un síndrome metabólico complejo con la singularidad de haber pérdida de músculo con o sin pérdida de grasa. La mayoría de pacientes caquéxicos son también sarcopénicos, pero no así a la inversa.

La fragilidad es un síndrome geriátrico que resulta del decaimiento de múltiples sistemas fisiológicos, con reserva homeostática disminuida y limitada capacidad para sobrellevar estrés. Esto los predispone a caídas, hospitalización, institucionalización, mortalidad, deterioro cognitivo, y baja red de apoyo. Se identifica por los siguientes elementos: pérdida de peso sin intención, debilidad, marcha lenta, baja actividad física y agotamiento (17). Existe un solape entre fragilidad y sarcopenia, la mayoría de los adultos mayores con fragilidad tienen sarcopenia lo cual sugiere con mecanismo fisiopatológico común.

La obesidad sarcopénica es una condición médica en la que disminución muscular vista en la sarcopenia se asocia a un aumento en la grasa. Tiene una prevalencia que va de $2 \%$ a $21,7 \%$, esta variabilidad se explica debido a falta de conocimiento de esta patología por los proveedores de salud, diferencias genéticas, nutrición y estilo de vida (18). La disminución de la masa muscular con aumento de la grasa es esperable en el 
proceso de envejecimiento. La presencia de obesidad sarcopénica es un reto diagnóstico debido a que la reducción muscular debido a la edad puede ser independiente del índice de masa corporal.

\section{CONCLUSIÓN}

Se debe tener alta sospecha clínica para diagnosticarla, por lo que se recomienda el uso de cuestionario SARC-F. El diagnostico se basa en comprobar mediante estudios de gabinete y examen físico la perdida de función y masa muscular. Cada vez se le da mayor énfasis a la valoración de la calidad del tejido muscular con herramientas como el TAC, US o RMN. Siempre se tiene que valorar el rendimiento físico para definir la severidad. El abordaje diagnostico mediante los 4 pasos es un método sencillo que ayudará al clínico a mantener un orden mental y valorar la patología desde un punto vista global.

Se han realizado estudios para valorar eficacia de biomarcadores que ayuden al diagnóstico temprano, pero no se ha encontrado ninguno con aplicabilidad clínica. La FDA no ha aceptado ningún fármaco especifico, pero si se hace énfasis en ejercicios de resistencia y dieta con mayor ingesta proteica.

\section{REFERENCIAS}

1. Dent E, Morley JE, Cruz-Jentoft AJ, Arai H, Kritchevsky SB, et al. International Clinical Practice Guidelines for Sarcopenia (ICFSR): Screening, Diagnosis and Management. The journal of nutrition, health \& aging. 2018 Nov 22; 22(10): 1148-1161. https://doi.org/10.1007/s12603-018-1139-9

2. Cruz-Jentoft AJ, Landi F, Schneider SM, Zuniga C, Arai H, et al. Prevalence of and interventions for sarcopenia in ageing adults: a systematic review. Report of the International Sarcopenia Initiative (EWGSOP and IWGS). Age and Ageing. 201409 21; 43(6): 748-759. https://doi.org/10.1093/ageing/afu115

3. Anker SD, Morley JE, von Haehling S. Welcome to the ICD-10 code for sarcopenia. Journal of Cachexia, Sarcopenia and Muscle. 2016 Oct 17; 7(5): 512-514. https://doi.org/10.1002/jcsm.12147

4. Dhillon RJ, Hasni S. Pathogenesis and Management of Sarcopenia. Clinics in Geriatric Medicine. 2017 02; 33(1): 17-26. https://doi.org/10.1016/i.cger.2016.08.002

5. Woo J. Sarcopenia. Clinics in Geriatric Medicine. 2017 08; 33(3): $305-314$. https://doi.org/10.1016/j.cger.2017.02.003.

6. Yu J. The etiology and exercise implications of sarcopenia in the elderly. International Journal of Nursing Sciences. 2015 06; 2(2): 199-203. https://doi.org/10.1016/j.ijnss.2015.04.010

7. Calvani R, Marini F, Cesari M, Tosato M, Anker SD, et al. Biomarkers for physical frailty and sarcopenia: state of the science and future developments. Journal of Cachexia, Sarcopenia and Muscle. 20150707 ; 6(4): 278-286.https://doi.org/10.1002/jcsm.12051

8. Cruz-Jentoft AJ, Bahat G, Bauer J, Boirie Y, Bruyère O, et al. Sarcopenia: revised European consensus on definition and diagnosis. Age and Ageing. 201809 24; 48(1): 16-31. https://doi.org/10.1093/ageing/afy169 
9. Malmstrom TK, Morley JE. SARC-F: A Simple Questionnaire to Rapidly Diagnose Sarcopenia. Journal of the American Medical Directors Association. 2013 08; 14(8): 531532. https://doi.org/10.1016/i.jamda.2013.05.018

10. Beaudart $\mathrm{C}, \mathrm{McCl}$ coskey $\mathrm{E}$, Bruyère $\mathrm{O}$, Cesari $\mathrm{M}$, Rolland $\mathrm{Y}$, et al. Sarcopenia in daily practice: assessment and management. BMC Geriatrics. 2016 Oct 05; 16: 170. https://doi.org/10.1186/s12877-016-0349-4

11. Lee D, Shook RP, Drenowatz C, Blair SN. Physical activity and sarcopenic obesity: definition, assessment, prevalence and mechanism. Future Science OA. 2016 09; 2(3): FSO127. https://doi.org/10.4155/fsoa-2016$\underline{0028}$

12. Yarasheski KE. Exercise, Aging, and Muscle Protein Metabolism. The Journals of Gerontology Series A. 2003 Oct 01; 58(10): M918-M922. https://doi.org/10.1093/gerona/58.10.m918

13. Roth SM, Ferrell RF, Hurley BF. Strength training for the prevention and treatment of sarcopenia. The Journal of Nutrition Health and Aging. 2000; 4(3): 143-155. Disponible en: https://www.ncbi.nlm.nih.gov/pubmed/10936901

14. Phillips SM, Chevalier S, Leidy HJ. Protein "requirements" beyond the RDA: implications for optimizing health. Applied Physiology, Nutrition, and Metabolism. 2016 05; 41(5): 565572. https://doi.org/10.1139/apnm-2015-0550

15. Stewart Coats AJ, Srinivasan V, Surendran J, Chiramana H, Vangipuram SRKG, et al. The ACT-ONE trial, a multicentre, randomised, double-blind, placebo-controlled, dose-finding study of the anabolic/catabolic transforming agent, MT-102 in subjects with cachexia related to stage III and IV non-small cell lung cancer and colorectal cancer:. Journal of Cachexia, Sarcopenia and Muscle. 2011 Oct 16; 2(4): 201207. https://doi.org/10.1007/s13539-011-0046-2

16. Burks TN, Andres-Mateos E, Marx R, Mejias R, Van Erp C, et al. Losartan Restores Skeletal Muscle Remodeling and Protects Against Disuse Atrophy in Sarcopenia. Science Translational Medicine. 201105 11; 3(82): 82ra37-82ra37.https://doi.org/10.1126/scitranslmed.3002227

17. Clegg A, Young J, lliffe S, Rikkert MO, Rockwood K. Frailty in elderly people. The Lancet. 201303 ; 381(9868): 752-762. https://doi.org/10.1016/s0140-6736(12)62167-9

18. Waters DL, Baumgartner RN. Sarcopenia and Obesity. Clinics in Geriatric Medicine. 2011 08; 27(3): 401421.https://doi.org/10.1016/i.cger.2011.03.007 